OU-HET 218

YITP/U-95-29

August, 1995

\title{
Macroscopic Three-Loop Amplitudes and the Fusion Rules from the Two-Matrix Model \&
}

\author{
M. Anazawa ${ }^{\dagger}$ Z, A. Ishikawa ${ }^{\ddagger 2}$ \\ and \\ H. Itoyama ${ }^{\dagger}$ \\ $\dagger$ Department of Physics, \\ Faculty of Science, Osaka University, \\ Toyonaka, Osaka, 560 Japan \\ and \\ ‡ Uji Research Center, Yukawa Institute for Theoretical Physics, \\ Kyoto University, Uji, Kyoto, 611 Japan
}

\begin{abstract}
From the computation of three-point singlet correlators in the two-matrix model, we obtain an explicit expression for the macroscopic three-loop amplitudes having boundary lengths $\ell_{i}(i=1 \sim 3)$ in the case of the unitary series $(p, q)=(m+1, m)$ coupled to two-dimensional gravity. The sum appearing in this expression is found to conform to the structure of the CFT fusion rules while the summand factorizes through a product of three modified Bessel functions. We briefly discuss a possible generalization of these features to macroscopic $n$-loop amplitudes.
\end{abstract}

\footnotetext{
${ }^{1}$ This work is supported in part by Grant-in-Aid for Scientific Research (07640403) and by the Grand-in-Aid for Scientific Research Fund $(2690,5108)$ from the Ministry of Education, Science and Culture, Japan.

${ }^{2}$ JSPS fellow
} 
The macroscopic $n$-loop amplitudes describe fundamental processes of $c \leq 1$ noncritical strings. Their expression is well-known in the case of pure two-dimensional gravity realized as the critical point of the one-matrix model [1]: it takes a suggestive form that the product of factors appears as external legs and the rest are expressible in terms of the sum of the total lengths. These seem to summarize the lore of the noncritical string theory in less than one dimension. No comparable expression is known for the case of the unitary minimal models coupled to gravity [1], [2]. In the previous letter [3], however, we have obtained the two-loop (annulus) amplitude having the boundary lengths $\ell_{1}$ and $\ell_{2}$ from the planar solution of the two-matrix model for the case of the unitary series; $(p, q)=(m+1, m)$ [4] (See [1], [5], [6] for the case of the one-matrix model). Let us first recall this amplitude: ${ }^{3 \text {, f }}$

$$
w\left(\ell_{1}, \ell_{2}\right)_{c}=\frac{2}{m a^{2} \pi^{2}} \frac{M \ell_{1} \ell_{2}}{\ell_{1}+\ell_{2}} \sum_{k=1}^{m-1}\left(\sin \frac{k}{m} \pi\right)^{2} K_{\frac{k}{m}}\left(M \ell_{1}\right) K_{1-\frac{k}{m}}\left(M \ell_{2}\right),
$$

where $M=t^{1 / 2}$ and $t$ is the renormalized cosmological constant (The definition of $a$ and related objects will be given later in eq. (18), (19).) f. The product of the modified Bessel functions multiplied by the length $M \ell_{i} K_{\nu}\left(M \ell_{i}\right)$ is naturally interpreted as external leg factors (wave functions). This, together with the absence of a propagator, will constitute integral parts of Feynman-like rules of noncritical $c \leq 1$ string 5 . It is also tempting to regard the integer $k$ summed as a label for diagonal primary states of underlying CFT, which is now gravitationally dressed.

In this letter, we will corroborate these features further: we present an explicit expression for macroscopic three-loop amplitudes in the case of the unitary minimal models coupled to two-dimensional gravity. We obtain this from the computation of correlators consisting of the product of three resolvents in the two-matrix model at the $m$-th critical point. We first state our expression for the three-loop $w\left(\ell_{1}, \ell_{2}, \ell_{3}\right)_{c}$ which is manifestly symmetric under the interchange $\ell_{i} \leftrightarrow \ell_{j}$ :

$$
w\left(\ell_{1}, \ell_{2}, \ell_{3}\right)_{c}=-\frac{1}{m(m+1) a^{3} \pi^{3}}\left(\frac{a M}{2}\right)^{-2-1 / m}
$$

\footnotetext{
3 This amplitude is for the case in which the spins on the two-boundaries are oriented in the same direction. The case of the opposite direction is obtained by replacing $\ell_{1}+\ell_{2}$ by $\ell_{1}+(-)^{m} \ell_{2}$ and by inserting $(-)^{k}$ in the summand.

${ }^{4}$ We have discussed some correspondence of this amplitude with the one from the continuum approach (see also [7]).

${ }^{5} \mathrm{~A}$ similar but distinct formula is seen in 8$]$.

${ }^{6}$ Note that the disk-amplitude is also proportinal to $\left(\sin \frac{\pi}{m}\right) \frac{M^{1+1 / m}}{\ell} K_{1+1 / m}(M \ell)$.
} 


$$
\begin{aligned}
& \times \sum_{\mathcal{D}_{3}\left(k_{1}-1, k_{2}-1, k_{3}-1\right)} \sin \frac{k_{1}}{m} \pi \sin \frac{k_{2}}{m} \pi \sin \frac{k_{3}}{m} \pi \\
& \times \quad M^{3} \ell_{1} \ell_{2} \ell_{3} K_{1-\frac{k_{1}}{m}}\left(M \ell_{1}\right) K_{1-\frac{k_{2}}{m}}\left(M \ell_{2}\right) K_{1-\frac{k_{3}}{m}}\left(M \ell_{3}\right) .
\end{aligned}
$$

Here we have denoted by $\mathcal{D}_{3}$

$$
\begin{aligned}
\mathcal{D}_{3}\left(a_{1}, a_{2}, a_{3}\right)=\left\{\left(a_{1}, a_{2}, a_{3}\right) \mid \sum_{i=1}^{3} a_{i}\right. & \leq 2(m-2), \quad \sum_{i=1}^{3} a_{i}=\text { even } \\
0 & \left.\leq\left(\sum_{i(\neq j)}^{3} a_{i}\right)-a_{j}, \quad j=1 \sim 3\right\}
\end{aligned}
$$

As one may have expected, the product of three factors $\left(\sin \frac{k_{i}}{m} \pi\right) M \ell_{i} K_{\nu}\left(M \ell_{i}\right)$ have appeared.

It is interesting that the selection rules obtained from $\mathcal{D}_{3}$ for the set of integers coincide with the fusion rules of the underlying conformal field theory of the unitary minimal series $(m+1, m)$ [9]. In fact, the fusion rules for diagonal primary fields read as

$$
\left\langle\phi_{i i} \phi_{j j} \phi_{k k}\right\rangle \neq 0
$$

if and only if $i+j \geq k+1$ and two other permutations and $i+j+k(=$ odd $) \leq 2 m-1$ hold. This set of rules is nothing but $\mathcal{D}_{3}(i-1, j-1, k-1)$.

This coincidence can be understood a little better by studying the small length behavior of eq. (2). By letting $M \ell_{i}<<1$ independently, we find that eq. (2) becomes proportional to

$$
\left(\frac{a M}{2}\right)^{-2-1 / m} \sum_{\mathcal{D}_{3}\left(k_{1}-1, k_{2}-1, k_{3}-1\right)} M^{\frac{\sum k_{i}}{m}} \ell_{1}^{k_{1} / m} \ell_{2}^{k_{2} / m} \ell_{3}^{k_{3} / m} 2^{3-\frac{\sum k_{i}}{m}}
$$

where we have used the formula

$$
K_{\nu}(x)=\frac{\pi}{2 \sin (\nu \pi)}\left[\frac{1}{x^{\nu}}\left\{\frac{2^{\nu}}{\Gamma(1-\nu)}+O\left(x^{2}\right)\right\}+x^{\nu}\left\{-\frac{1}{2^{\nu} \Gamma(1+\nu)}+O\left(x^{2}\right)\right\}\right] .
$$

The $M$-dependence of the individual terms in eq. (5) is $M^{-2+\frac{\left(\sum k_{i}\right)-1}{m}}$. This is compared with the computation done in ref. [10] (See ref. [11] from the point of view of the continuum approach.) from the generalized Kdv flows for the correlation functions of the dressed primaries:

$$
\left\langle\phi_{\ell} \phi_{p} \phi_{r}\right\rangle=\operatorname{lpr}\left(\frac{u}{2}\right)^{(\ell+p+r-3) / 2} u^{\prime}
$$


Under $u\left(=t^{1 / m}\right)$, the $t$-dependence of this expression completely agrees with that of eq. (5). The selection rules for this expression found in ref. 10 is again summarized as $\mathcal{D}_{3}$.

Our formula tells how the higher order operators (gravitational descendants) in addition to the dressed primaries are constrained to obey the selection rules of CFT. Let us remark here that the $c=1$ limit can be studied from our formula [2]. We have checked that, by letting one of the three lengths shrink $\left(M \ell_{i}<<1\right)$, the three-loop amplitude reduces to the derivative of the two-loop amplitude with respect to the cosmological constant $t$ up to a normalization factor.

The remainder of this letter is devoted to the derivation of eq. (2) from the twomatrix model at the $(m+1, m)$ critical point. We will also briefly discuss what in eq. (2) may be generalized to the expression for macroscopic $n$-loop amplitudes.

To begin with let us consider the connected three-point singlet correlator consisting of arbitrary analytic functions $f(\hat{A}), g(\hat{A})$, and $h(\hat{A})$, where the matrix $\hat{A}$ is a dynamical variable. In the bases of orthogonal polynomials, one can derive

$$
\begin{aligned}
\langle\operatorname{Tr} f(\hat{A}) \operatorname{Tr} g(\hat{A}) \operatorname{Tr} h(\hat{A})\rangle_{c} & =\sum_{i=0}^{N-1} \sum_{k=N}^{\infty} \sum_{l=N}^{\infty}[f(A)]_{i k}[g(A)]_{k l}[h(A)]_{l i} \\
& -\sum_{i=0}^{N-1} \sum_{k=N}^{\infty} \sum_{l=0}^{N-1}[f(A)]_{i k}[h(A)]_{k l}[g(A)]_{l i},
\end{aligned}
$$

where $[f(A)]_{i k} \equiv\langle i|f(\lambda)| k\rangle$. It is convenient to introduce the following 'classical' function in the case only the planar limit is of interest,

$$
A(z, s, \Lambda) \equiv \sum_{k} z^{k} A(i)_{k} \quad, \quad s \equiv \Lambda i / N \quad
$$

where $A(i)_{k} \equiv A_{i-k, i}$. The 'classical' function depends on the bare cosmological constant $\Lambda$ only through $s$ when we take $N \rightarrow \infty$ limit. It is, therefore, legitimate to introduce $A(z, s) \equiv \lim _{N \rightarrow \infty} A(z, s, \Lambda)$ :

$$
A(z, s, \Lambda)=A(z, s)+O(1 / N)
$$

In terms of the 'classical' function, we have

$$
[f(A)](N)_{k}=\frac{1}{2 \pi i} \oint \frac{\mathrm{d} z}{z^{k+1}} f(A(z, s=\Lambda))+O(1 / N),
$$

where $[f(A)](i)_{k} \equiv[f(A)]_{i-k, i}$. In the right-hand sind of eq. (8), the leading terms in $1 / N$ of the first term and those of the second term get cancelled. We have to consider 
the next leading terms. For any integer $\epsilon$, we obtain

$$
\begin{aligned}
{[f(A)](N+\epsilon)_{k} } & =\frac{1}{2 \pi i} \oint \frac{\mathrm{d} z}{z^{k+1}} f(A(z, s=\Lambda)) \\
& +\left.\frac{\Lambda \epsilon}{N} \frac{1}{2 \pi i} \oint \frac{\mathrm{d} z}{z^{k+1}} \frac{\partial A(z, s)}{\partial s}\right|_{s=\Lambda} \frac{\partial f(A(z, \Lambda))}{\partial A} \\
& + \text { (the part independent of } \epsilon)+O\left(1 / N^{2}\right)
\end{aligned}
$$

The part independent of $\epsilon$ comes from the terms $O(1 / N)$ in eq. (11). The second term is responsible for the computation in what follows. Using eq. (12) and considering the terms $1 / N$ in eq. (8), we obtain

$$
\begin{aligned}
&\langle\operatorname{Tr} f(\hat{A}) \operatorname{Tr} g(\hat{A}) \operatorname{Tr} h(\hat{A})\rangle_{c} \\
&=\frac{\Lambda}{N} \sum_{\delta_{1}=0}^{\infty} \sum_{\delta_{2}=0}^{\infty} \sum_{\delta=0}^{\infty} \\
& \quad\left\{\left(\delta_{2}-\delta_{1}\right) \frac{\partial}{\partial s}[f(A)](N)_{\delta_{1}+\delta_{2}+1}[g(A)](N)_{\delta-\delta_{2}}[h(A)](N)_{-\delta-\delta_{1}-1}\right. \\
&+\quad\left(\delta+\delta_{2}+1\right)[f(A)](N)_{\delta_{1}+\delta_{2}+1} \frac{\partial}{\partial s}[g(A)](N)_{\delta-\delta_{2}}[h(A)](N)_{-\delta-\delta_{1}-1} \\
&\left.+\quad\left(\delta-\delta_{1}\right)[f(A)](N)_{\delta_{1}+\delta_{2}+1}[g(A)](N)_{\delta-\delta_{2}} \frac{\partial}{\partial s}[h(A)](N)_{-\delta-\delta_{1}-1}\right\} \\
&+ O\left(1 / N^{2}\right) .
\end{aligned}
$$

Using eq. (11) the three-point function eq. (8) in the planar limit can be expressed in terms of the 'classical' function as

$$
\begin{aligned}
\langle\operatorname{Tr} & f(\hat{A}) \operatorname{Tr} g(\hat{A}) \operatorname{Tr} h(\hat{A})\rangle_{c} \\
= & \frac{\Lambda}{N} \frac{1}{(2 \pi i)^{3}} \oint_{|z|>\left|z^{\prime}\right|>\left|z^{\prime \prime}\right|} \mathrm{d} z \mathrm{~d} z^{\prime} \mathrm{d} z^{\prime \prime} \\
& \left\{\frac{z}{\left(z-z^{\prime}\right)^{2}\left(z-z^{\prime \prime}\right)^{2}} f^{\prime}(A(z)) \frac{\partial A(z)}{\partial \Lambda} g\left(A\left(z^{\prime}\right)\right) h\left(A\left(z^{\prime \prime}\right)\right)\right. \\
+ & \frac{z^{\prime}}{\left(z-z^{\prime}\right)^{2}\left(z^{\prime}-z^{\prime \prime}\right)^{2}} f(A(z)) g^{\prime}\left(A\left(z^{\prime}\right)\right) \frac{\partial A\left(z^{\prime}\right)}{\partial \Lambda} h\left(A\left(z^{\prime \prime}\right)\right) \\
+ & \left.\frac{z^{\prime \prime}}{\left(z-z^{\prime \prime}\right)^{2}\left(z^{\prime}-z^{\prime \prime}\right)^{2}} f(A(z)) g\left(A\left(z^{\prime}\right)\right) h^{\prime}\left(A\left(z^{\prime \prime}\right)\right) \frac{\partial A\left(z^{\prime \prime}\right)}{\partial \Lambda}\right\},
\end{aligned}
$$

where we set $A(z) \equiv A(z, s=\Lambda)$. From the above formula (eq. (14)), the three-point resolvent in the planar limit is expressed as

$$
\frac{N}{\Lambda}\left\langle\operatorname{Tr} \frac{1}{p_{1}-\hat{A}} \operatorname{Tr} \frac{1}{p_{2}-\hat{A}} \operatorname{Tr} \frac{1}{p_{3}-\hat{A}}\right\rangle_{c}
$$




$$
\begin{aligned}
= & \frac{1}{(2 \pi i)^{3}} \oint_{|z|>\left|z^{\prime}\right|>\left|z^{\prime \prime}\right|} \mathrm{d} z \mathrm{~d} z^{\prime} \mathrm{d} z^{\prime \prime} \\
& \left\{\frac{z}{\left(z-z^{\prime}\right)^{2}\left(z-z^{\prime \prime}\right)^{2}} \frac{\partial A(z)}{\partial \Lambda} \frac{1}{\left[p_{1}-A(z)\right]^{2}} \frac{1}{p_{2}-A\left(z^{\prime}\right)} \frac{1}{p_{3}-A\left(z^{\prime \prime}\right)}\right. \\
+ & \frac{z^{\prime}}{\left(z-z^{\prime}\right)^{2}\left(z^{\prime}-z^{\prime \prime}\right)^{2}} \frac{1}{p_{1}-A(z)} \frac{\partial A\left(z^{\prime}\right)}{\partial \Lambda} \frac{1}{\left[p_{2}-A\left(z^{\prime}\right)\right]^{2}} \frac{1}{p_{3}-A\left(z^{\prime \prime}\right)} \\
+ & \left.\frac{z^{\prime \prime}}{\left(z-z^{\prime \prime}\right)^{2}\left(z^{\prime}-z^{\prime \prime}\right)^{2}} \frac{1}{p_{1}-A(z)} \frac{1}{p_{2}-A\left(z^{\prime}\right)} \frac{\partial A\left(z^{\prime \prime}\right)}{\partial \Lambda} \frac{1}{\left[p_{3}-A\left(z^{\prime \prime}\right)\right]^{2}}\right\},
\end{aligned}
$$

where the contour of $z$ encircles that of $z^{\prime}$ and similarly the contour of $z^{\prime}$ encircles that of $z^{\prime \prime}$. We calculate these contour integrals and find

$$
\begin{aligned}
\frac{N}{\Lambda}\left\langle\operatorname{Tr} \frac{1}{p_{1}-\hat{A}}\right. & \left.\operatorname{Tr} \frac{1}{p_{2}-\hat{A}} \operatorname{Tr} \frac{1}{p_{3}-\hat{A}}\right\rangle_{c} \\
= & -\frac{1}{a^{3}}\left\{\frac{\partial z_{1}}{\partial \Lambda} \frac{\partial}{\partial z_{1}}\left[\frac{z_{1}}{\left(z_{1}-z_{2}\right)^{2}\left(z_{1}-z_{3}\right)^{2}} \frac{\partial z_{1}}{\partial \zeta_{1}}\right] \frac{\partial z_{2}}{\partial \zeta_{2}} \frac{\partial z_{3}}{\partial \zeta_{3}}\right. \\
& +\frac{\partial z_{1}}{\partial \zeta_{1}} \frac{\partial z_{2}}{\partial \Lambda} \frac{\partial}{\partial z_{2}}\left[\frac{z_{2}}{\left(z_{1}-z_{2}\right)^{2}\left(z_{2}-z_{3}\right)^{2}} \frac{\partial z_{2}}{\partial \zeta_{2}}\right] \frac{\partial z_{3}}{\partial \zeta_{3}} \\
& \left.+\frac{\partial z_{1}}{\partial \zeta_{1}} \frac{\partial z_{2}}{\partial \zeta_{2}} \frac{\partial z_{3}}{\partial \Lambda} \frac{\partial}{\partial z_{3}}\left[\frac{\partial z_{3}}{\left(z_{1}-z_{3}\right)^{2}\left(z_{2}-z_{3}\right)^{2}} \frac{\partial \zeta_{3}}{\partial \zeta_{3}}\right]\right\}
\end{aligned}
$$

where

$$
\begin{gathered}
z_{i}=\exp \left(2 \eta \cosh \theta_{i}\right) \\
p_{i}-p_{*}=a \zeta_{i}=2 \eta^{m} \cosh m \theta_{i}=a M \cosh m \theta_{i} \quad, \quad \eta=(a M / 2)^{1 / m} \\
\Lambda-\Lambda_{*}=-(m+1) \eta^{2 m}=-(m+1) a^{2} \mu=-(m+1)(a M / 2)^{2}
\end{gathered}
$$

and $p_{*}, \Lambda_{*}$ denote the critical values of $p, \Lambda$ respectively. The right hand side of eq. (16) can be written in a compact form as

$$
\begin{aligned}
\frac{N}{\Lambda}\left\langle\operatorname{Tr} \frac{1}{p_{1}-\hat{A}}\right. & \left.\operatorname{Tr} \frac{1}{p_{2}-\hat{A}} \operatorname{Tr} \frac{1}{p_{3}-\hat{A}}\right\rangle_{c} \\
& =\frac{1}{2^{2} m(m+1) a^{3}}\left(\frac{a M}{2}\right)^{-2-1 / m} \frac{\partial}{\partial \zeta_{1}} \frac{\partial}{\partial \zeta_{2}} \frac{\partial}{\partial \zeta_{3}} F\left(\theta_{1}, \theta_{2}, \theta_{3}\right)
\end{aligned}
$$

where

$$
\begin{aligned}
F\left(\theta_{1}, \theta_{2}, \theta_{3}\right) & =\frac{1}{\left(\cosh \theta_{1}-\cosh \theta_{2}\right)\left(\cosh \theta_{1}-\cosh \theta_{3}\right)} \frac{\sinh (m-1) \theta_{1}}{\sinh m \theta_{1}} \\
& +(1 \leftrightarrow 2)+(1 \leftrightarrow 3)
\end{aligned}
$$


Making use of the following formula twice,

$$
\begin{gathered}
\frac{1}{\cosh \alpha-\cosh \beta}\left(\frac{\sinh (n-k) \alpha}{\sinh n \alpha}-\frac{\sinh (n-k) \beta}{\sinh n \beta}\right) \\
=-2 \sum_{j=1}^{n-k} \sum_{i=1}^{k} \frac{\sinh (n-j-i+1) \alpha}{\sinh n \alpha} \frac{\sinh (n-j-k+i) \beta}{\sinh n \beta}
\end{gathered}
$$

we find that eq. (21) is written as a triple sum where the summand factorizes into three factors associated with individual loops:

$$
\begin{aligned}
& F\left(\theta_{1}, \theta_{2}, \theta_{3}\right) \\
& \quad=4 \sum_{k=1}^{m-1} \sum_{j=1}^{m-k} \sum_{i=1}^{k} \frac{\sinh (m-k) \theta_{1}}{\sinh m \theta_{1}} \frac{\sinh (m-j-i+1) \theta_{2}}{\sinh m \theta_{2}} \frac{\sinh (m-k-j+i) \theta_{3}}{\sinh m \theta_{3}}
\end{aligned}
$$

Here, $W\left(\zeta_{1}, \zeta_{2}, \zeta_{3}\right)_{c}=\frac{N}{\Lambda}\left\langle\operatorname{Tr} \frac{1}{p_{1}-\hat{A}} \operatorname{Tr} \frac{1}{p_{2}-\hat{A}} \operatorname{Tr} \frac{1}{p_{3}-\hat{A}}\right\rangle_{c}$ and $w\left(\ell_{1}, \ell_{2}, \ell_{3}\right)_{c}$ are related by the Laplace transform

$$
\begin{aligned}
W\left(\zeta_{1}, \zeta_{2}, \zeta_{3}\right)_{c} & =\int_{0}^{\infty} d \ell_{1} \int_{0}^{\infty} d \ell_{2} \int_{0}^{\infty} d \ell_{3} e^{-\zeta_{1} \ell_{1}} e^{-\zeta_{2} \ell_{2}} e^{-\zeta_{2} \ell_{3}} w\left(\ell_{1}, \ell_{2}, \ell_{3}\right)_{c} \\
& \equiv \mathcal{L}\left[w\left(\ell_{1}, \ell_{2}, \ell_{3}\right)_{c}\right] .
\end{aligned}
$$

In [3], we have found the following formula for the inverse Laplace image

$$
\mathcal{L}^{-1}\left[\frac{\partial}{\partial \zeta} \frac{\sinh k \theta}{\sinh m \theta}\right]=-\frac{M \ell}{\pi} \sin \frac{k \pi}{m} K_{\frac{k}{m}}(M \ell)
$$

Note that $K_{\nu}(z)$ is the modified Bessel function.

Inverse Laplace transform of eq. (24) gives the three-loop amplitude in terms of each boundary length. We obtain

$$
\begin{aligned}
w\left(\ell_{1}, \ell_{2}, \ell_{3}\right)_{c} & =-\frac{1}{m(m+1) a^{3} \pi^{3}}\left(\frac{a M}{2}\right)^{-2-1 / m} \\
& \times \sum_{k=1}^{m-1} \sum_{j=1}^{m-k} \sum_{i=1}^{k} \sin \frac{m-k}{m} \pi \sin \frac{m-j-i+1}{m} \pi \sin \frac{m-k-j+i}{m} \pi \\
& \times M^{3} \ell_{1} \ell_{2} \ell_{3} K_{\frac{m-k}{m}}\left(M \ell_{1}\right) K_{\frac{m-j-i+1}{m}}\left(M \ell_{2}\right) K_{\frac{m-k-j+i}{m}}\left(M \ell_{3}\right) .
\end{aligned}
$$

Let us write the set of restrictions on the triple sum in eq. (26) as

$$
\mathcal{F}(k, j, i)=\{(k, j, i) \mid 1 \leq k \leq m-1,1 \leq j \leq m-k, 1 \leq i \leq k\}
$$


By elementary algebras, one can show

$$
\begin{aligned}
\mathcal{F}(k, j, i) & =\mathcal{D}_{3}\left(k_{1}-1, k_{2}-1, k_{3}-1\right) \\
& =\mathcal{D}_{3}(k-1,(j+i-1)-1,(k+j-i)-1)
\end{aligned}
$$

This completes the derivation of eq. (21).

Finally, let us briefly discuss how the features of the three-loop amplitude we have found here may be generalized to the $n$-loop amplitudes. It is for sure that the summand contains a product of $n$-external leg factors $\left(\sin \frac{k_{i}}{m} \pi\right) M \ell_{i} K_{1-\frac{k_{i}}{m}}\left(M \ell_{i}\right)$ $(i=1 \sim m)$ but it must be supplemented by a symmetric polynomial, which we need to determine. As for the restrictions on the sum, let us first note that $\mathcal{D}_{3}$, which is fusion rules, is rephrased as the condition that a triangle with edges $\left(a_{1}, a_{2}, a_{3}\right)$ be formed whose circumference is even and less than or equal to $2(m-2)$. The $n$-loop amplitudes must respect the fusion rules and these triangles will become building blocks for the case of $n$-loop amplitudes. The $n$-gons made out of $n-2$ triangles of this type

$$
\begin{aligned}
\mathcal{D}_{n}\left(a_{1}, \ldots, a_{n}\right) & =\left\{\left(a_{1}, \ldots, a_{n}\right) \mid \sum_{i=1}^{n} a_{i}=\text { even },\right. \\
0 & \left.\leq\left(\sum_{i \neq j}^{n} a_{i}\right)-a_{j}, \quad j=1 \sim n\right\}
\end{aligned}
$$

are relevant. Different shapes of $n$-gons for given $\left(a_{1}, \ldots, a_{n}\right)$ and various different divisions of each $n$-gon are important ingredients of the completely and crossing symmetric properties of the amplitudes. Also note that the restriction on the sum in the case of the two-loop (annulus) amplitudes is written as $\mathcal{D}_{2}\left(m-k_{1}, k_{2}\right)$ under the convention of the factors $M^{2} \ell_{1} \ell_{2} K_{1-\frac{k_{1}}{m}}\left(M \ell_{1}\right) K_{1-\frac{k_{2}}{m}}\left(M \ell_{2}\right)$. Despite these prominent features, complete determination of the amplitudes still requires computation from the two-matrix model. 


\section{References}

[1] G. Moore, N. Seiberg and M. Staudacher, Nucl. Phys. B362 (1991) 665.

[2] G. Moore and N. Seiberg, Int. J. Mod. Phys. A7 (1992) 2601.

[3] M. Anazawa, A. Ishikawa and H. Itoyama, preprint OU-HET 190, YITP/U-9434, hep-th/9410015, to appear in Phys. Rev. D.

[4] J.M. Daul, V.A. Kazakov and I.K. Kostov, Nucl. Phys. B409 (1993) 311.

[5] T. Banks, M. Douglas, N. Seiberg and S. Shenker, Phys. Lett. B238 (1990) 279.

[6] L. Alvarez-Gaumé, H. Itoyama, J.L. Manes and A. Zadra, Int. J. Mod. Phys. A7 (1992) 5337.

[7] R. Nakayama, Phys. Lett. B325 (1993) 347.

[8] I. K. Kostov, Nucl. Phys. B376 (1992) 539.

[9] A. Belavin, A.M. Polyakov and A.B. Zamolodchikov, Nucl. Phys. B241 (1984) 333.

[10] P. DiFrancesco and D. Kutasov, Nucl. Phys. B342 (1990) 589.

[11] L. Alvarez-Gaume, J. Barbon and C. Gomez, Nucl. Phys. B368 (1992) 57. 